\section{Using patient-centered end-points in older adults participating in clinical trials}

\author{
Arduino A. Mangoni, Kimberley Ruxton \\ Department of Clinical Pharmacology, \\ School of Medicine, Flinders University \\ and Flinders Medical Centre, Adelaide, \\ Australia
}

\begin{abstract}
Conventional end-points, primarily based on the pharmacodynamic effects of a specific drug, are used to assess the efficacy of pharmacological treatment in clinical trials. However, their application and interpretation in complex frail older patients, a patient group with high inter-individual variability, multiple coexisting disease states and prescribed medications, is becoming increasingly questionable. National surveys and qualitative studies have convincingly shown that the maintenance of functional independence is key to self-rated health and well being in old age. Therefore, the use of unconventional, patientcentered, end-points focused on functional status and perceived health seems appropriate, in combination with conventional end-points, to comprehensively investigate the impact of pharmacological treatments in this patient group. The recent availability of objective, quantifiable, and robust scoring tools, such as the multidimensional prognostic index, to assess key functional domains and clinical outcomes offers a unique opportunity to adequately characterize patient-centered endpoints in future clinical trials in older patients.
\end{abstract}

The progressive ageing of the population worldwide is considered a success story in terms of improved socioeconomic conditions as well as advances in the diagnosis and management of acute and chronic disease states. However, this socio-demographic change carries significant short- and long-term risks for the overall capacity and financial sustainability of health care systems in most countries. A key issue in this context is the increasing prevalence of older people with various degrees of disability and functional dependence, often the consequence of disease states that do not directly result in death. In particular, care transitions from a state of full independence in the community to dependence in one on more activities of daily living, but still in the community, to transfer into a nursing home or other aged care facilities, all impose a significant financial burden to the society. ${ }^{1}$ Several surveys and qualitative studies have also shown that the loss of independence is of major concern amongst older people living in the community, and their caregivers.,3 Therefore, the maintenance of adequate physical and cognitive function is pivotal to the perceived health and well being of older adults.

Although the loss of independence and disability in the older population are primarily disease-related, there is increasing evidence that the prescribing long-term of specific drugs and drug classes can also adversely affect physical and cognitive function in this group. For example, a number of observational studies have recently demonstrated that drugs with anticholinergic and/or sedative effects exert a negative impact on several activities of daily living, risk of falls and cognitive function in older adults. ${ }^{4-10}$ Therefore, the monitoring of physical and cognitive function, hence a state of independence, is not only important for older patients, and their relatives and caregivers, but also for assessing the effects of pharmacological interventions in this group. However, this concept fits poorly with the standard approach used to investigate the effects of pharmacological therapies in clinical trials. This primarily involves the assessment of conventional clinical end-points, e.g., blood pressure or serum cholesterol concentrations, based on the known pharmacodynamic effects of the studied drugs (Table 1). The use of conventional end-points has doubtlessly resulted in significant advances resulting in the publication of national and international guidelines on the management of numerous disease states. However, their application for prognostic and monitoring purposes is becoming increasingly questionable in the older population, particularly in complex frail older patients with multi-morbidity, polypharmacy, and relatively short life expectancy. Notably, this patient group is virtually neglected in Phase IIIII trials of new medicines, primarily because of the stringent inclusion and exclusion criteria and intensive follow-up assessments. The latter are more suitable for a younger and healthier participant population. ${ }^{11}$

Therefore, the generalizability of the results obtained from these trials to a patient population characterized by significant inter-individual differences in homeostatic reserve, pharmacokinetics and pharmacodynamics is problematic. ${ }^{12,13}$ This issue is further compounded by the potential therapeutic futility and offlabel prescribing of many medicines in this group. ${ }^{14}$ For these reasons, there is an urgent need to generate solid evidence in relation to the efficacy and safety of pharmacological therapies in complex frail older patients. A potential step forward in this context is the identification of unconventional, patient-centered, end-points specifically focused on meas-
Correspondence: Arduino A. Mangoni, Department of Clinical Pharmacology, School of Medicine, Flinders University and Flinders Medical Centre, Flinders Drive, Bedford Park, SA 5042, Australia. Tel: +61.8.8204.7495.

E-mail: arduino.mangoni@flinders.edu.au

Key words: Clinical trials; end-points; old age; drug development; frailty.

Contributions: AAM generated the idea and wrote the first draft; AAM and KR critically reviewed subsequent drafts and wrote the final version.

Conflict of interest: the authors declare no conflict of interest.

Conference presentation: this paper was presented at the International Symposium Anziano e far maci, $4^{\text {th }}$ Edition, Genoa, Italy, $11^{\text {th }}$ March 2016.

Received for publication: 21 March 2016.

Accepted for publication: 6 April 2016.

This work is licensed under a Creative Commons Attribution-NonCommercial 4.0 International License (CC BY-NC 4.0)

(C) Copyright A.A. Mangoni and K. Ruxton, 2016 Licensee PAGEPress, Italy

Geriatric Care 2016; 2:5893

doi:10.4081/sc.2016.5893

ures of perceived health and functional status (Table 1). Recent studies have elegantly shown that disease states with different pathophysiology, clinical presentation and overall prognosis exert a similar adverse impact on self-reported health and functional status in communitydwelling older patients. Moreover, the co-existence of two disease states was associated with a much greater adverse impact, when compared to a single pathological condition. ${ }^{15}$

Therefore, the use of patient-centered endpoints represents a promising strategy for better characterizing the effects of pharmacological interventions in old age. However, their assessment requires tools that are relatively easy to use in clinical practice, yet providing objective and quantifiable information to be used in clinical trials. A number of such tools have been developed to assess frailty and functional status in the older population. ${ }^{16}$ However, their applicability and predictive capacity in a naturalistic setting are uncertain. ${ }^{17}$ More recently, the multidimensional prognostic index (MPI), a quantifiable tool based on eight key domains of the comprehensive geriatric assessment, an established strategy to assess frailty and homeostatic capacity in order to formulate personalized care plans, has shown superior predictive capacity and discrimination in relation to adverse outcomes, when compared to other 
Table 1. Examples of conventional and patient-centered end-points.

\begin{tabular}{ll} 
Conventional end-points & Patient-centered end-points \\
Glycated hemoglobin & Fatigue \\
LDL-cholesterol concentrations & Weakness \\
\hline Blood pressure & Shortness of breath \\
Progression free survival & Activities of daily living \\
\hline Left ventricular ejection fraction & Dizziness \\
\hline
\end{tabular}

LDL, low-density lipoprotein.

established tools. The MPI has been assessed and validated in several cohort studies in older patients with different baseline clinical characteristics and functional status. ${ }^{18,19}$ Notably, the MPI has also been used as an end-point, to assess the effects of pharmacological and nonpharmacological treatment strategies in patients with dementia and depression. These studies have shown significant changes in the total MPI score during treatment. These changes suggested an improvement in different MPI domains, not limited to cognitive and behavioral domains..$^{20,21}$ These preliminary studies suggest that the MPI can also be used to assess the positive or negative effects of therapies on unconventional, patient-centered, end-points. ${ }^{22}$

What are the possible initial steps for a new approach to the assessment of the effects, positive and negative, of medications in the older population? Future phase II-III clinical trials should include, whenever possible, subgroups of frail older patients to identify early signs of efficacy and toxicity in this ever growing population. The inclusion of such patients would necessarily require some protocol adaptations, e.g., modified inclusion and exclusion criteria and reduced number of follow-up assessments. ${ }^{11}$ Moreover, studying established as well as unconventional end-points in these trials would allow a more comprehensive assessment of the benefits, risks, and potential futility of specific treatments. The availability of robust, easy to use, and quantifiable tools such as the MPI provides a unique opportunity for investigating these complex issues in this vulnerable group.

\section{References}

1. Guralnik JM, Alecxih L, Branch LG, Wiener JM. Medical and long-term care costs when older persons become more dependent. Am J Public Health 2002;92:1244-5.

2. Quine S, Morrell S. Fear of loss of independence and nursing home admission in older Australians. Health Soc Care Community 2007;15:212-20.

3. Bowling A, Gabriel Z, Dykes J, et al. Let's ask them: a national survey of definitions of quality of life and its enhancement among people aged 65 and over. Int J Aging Hum Dev 2003;56:269-306.

4. Bostock CV, Soiza RL, Mangoni AA. Association between prescribing of antimuscarinic drugs and antimuscarinic adverse effects in older people. Expert Rev Clin Pharmacol 2010;3:441-52.

5. Bostock CV, Soiza RL, Mangoni AA. Associations between different measures of anticholinergic drug exposure and Barthel Index in older hospitalized patients. Ther Adv Drug Saf 2013;4:235-45.

6. Koshoedo S, Soiza RL, Purkayastha R, Mangoni AA. Anticholinergic drugs and functional outcomes in older patients undergoing orthopaedic rehabilitation. Am J Geriatr Pharmacother 2012;10:251-7.

7. Lowry E, Woodman RJ, Soiza RL, et al. Drug burden index, physical function, and adverse outcomes in older hospitalized patients. J Clin Pharmacol 2012;52:1584-91.

8. Lowry E, Woodman RJ, Soiza RL, Mangoni AA. Associations between the anticholinergic risk scale score and physical function: potential implications for adverse outcomes in older hospitalized patients. J Am Med Dir Assoc 2011;12:565-72.

9. Mangoni AA. Assessing the adverse effects of antimuscarinic drugs in older patients: which way forward? Expert Rev Clin Pharmacol 2011;4:531-3.

10. Ruxton K, Woodman RJ, Mangoni AA. Drugs with anticholinergic effects and cognitive impairment, falls and all-cause mortality in older adults: a systematic review and meta-analysis. Br J Clin Pharmacol 2015;80:209-20.

11. Mangoni AA, Jansen PA, Jackson SH. Under-representation of older adults in pharmacokinetic and pharmacodynamic studies: a solvable problem? Expert Rev Clin Pharmacol 2013;6:35-9.

12. Mangoni AA, Jackson SH. Age-related changes in pharmacokinetics and pharmacodynamics: basic principles and practical applications. Br J Clin Pharmacol 2004; 57:6-14.

13. Reeve E, Wiese MD, Mangoni AA. Alterations in drug disposition in older adults. Expert Opin Drug Metab Toxicol 2015;11:491-508.

14. Jackson SH, Jansen PA, Mangoni AA. Offlabel prescribing in older patients. Drugs Aging 2012;29:427-34.

15. Tinetti ME, McAvay GJ, Chang SS, et al. Contribution of multiple chronic conditions to universal health outcomes. J Am Geriatr Soc 2011;59:1686-91.

16. Rodriguez-Manas L, Fried LP. Frailty in the clinical scenario. Lancet 2015;385:e7-9.

17. Deckx L, van den Akker M, Daniels L, et al. Geriatric screening tools are of limited value to predict decline in functional status and quality of life: results of a cohort study. BMC Fam Pract 2015;16:30.

18. Pilotto A, Rengo F, Marchionni N, et al. Comparing the prognostic accuracy for allcause mortality of frailty instruments: a multicentre 1-year follow-up in hospitalized older patients. PLoS One 2012;7: e29090.

19. Siontis GC, Tzoulaki I, Ioannidis JP. Predicting death: an empirical evaluation of predictive tools for mortality. Arch Intern Med 2011;171:1721-6.

20. D’Onofrio G, Sancarlo D, Addante F, et al. A pilot randomized controlled trial evaluating an integrated treatment of rivastigmine transdermal patch and cognitive stimulation in patients with Alzheimer's disease. Int J Geriatr Psychiatry 2015;30: 965-75.

21. Pilotto A, D’Onofrio G, Panza F, et al. Treatment of late-life major depressive disorder with selective serotonin reuptake inhibitors improves the multidimensional prognostic index. J Clin Psychopharmacol 2012;32:726-9.

22. Mangoni AA, Pilotto A. New drugs and patient-centred end-points in old age: setting the wheels in motion. Expert Rev Clin Pharmacol 2016;9:81-9. 